Article

\title{
Non-Linear Dynamic Feature Analysis of a Multiple-Stage Closed-Loop Gear Transmission System for 3D Circular Braiding Machine
}

\author{
Lingling Yao, Zhuo Meng *, Jianqiu Bu and Yize Sun \\ College of Mechanical Engineering, Donghua University, Shanghai 201620, China; \\ 1169168@mail.dhu.edu.cn (L.Y.); 1159156@mail.dhu.edu.cn (J.B.); sunyz@dhu.edu.cn (Y.S.) \\ * Correspondence: mz@dhu.edu.cn
}

Received: 29 September 2020; Accepted: 21 October 2020; Published: 28 October 2020

check for updates

\begin{abstract}
Aiming at the particularity of a multiple-stage closed-loop gear transmission system for 3D circular braiding machine, the model of gear transmission system in radial braiding machine was simplified. The non-linear dynamic equations of a n-elements closed-loop gear transmission system with symmetrical structure including static transmission error, the random disturbance of meshing damping and backlash were considered. For convenience of calculation $n=3$, the equations were solved numerically by using Runge-Kutta. The dynamic transmission error(DTE) with different backlash, dynamic meshing forces with and without the random disturbance of meshing damping, the amplitude of dynamic transmission error at $\mathrm{n}=1000 \mathrm{r} / \mathrm{min}$ and $\mathrm{b}=2.65 \times 10^{-5} \mathrm{~m}$, root mean square(RMS) of DTE and the mean value of DTE of the first pair of gears were analyzed. The simulation results show that different backlash and the random disturbance of meshing damping have a great influence on the dynamic displacement error and meshing force of the gear pair, and RMS and the mean value of DTE changes at different rotational speeds. The results will provide a reference for realizing the smoothness of the closed-loop gear transmission system with symmetrical structure for $3 \mathrm{D}$ braiding machine and have great practical significance for improving the braiding quality.
\end{abstract}

Keywords: 3D circular braiding machine; multiple-stage closed-loop gear transmission system; non-linear dynamic feature; the random disturbance of meshing damping; Runge-Kutta

\section{Introduction}

Braiding plays an important role in textile industry. It's very interesting for scholars to study braiding with the emergence of new materials and different types of braiding machines.

The performance analysis of composite materials, parameters, braiding process, and structure have been studied by scholars.Some works concerning the problem of corrosion in metallic materials treated with Swarm Optimization techniques and the work in which the problem of corrosion in composite materials is treated by means of acoustic techniques [1-3]. Reviews written by Guangli Ma et al. [4] proposed a method of tension versus yarn displacement. Haili Zhou, Wei Zhang et al. [5] analyzed the transverse impact behaviors. Guyader et al. [6] proposed the calculation method of process parameters. J.H. van Ravenhorst and Akkerman [7] proposed an inverse kinematics-based procedure to automatically generate machine control data. J.H. van Ravenhorst et al. [8] proposed a new method to simulate yarn interaction behavior. Won-Jin Na et al. [9] proposed a mathematical model to predict braid pattern. Hans et al. [10] simulated braiding process and experimented by the industrial robot. Kyosev [11] mentioned the radial braiding machine with a robot arm as a take-off device. Monnot et al. [12] researched automatic braiding using a non-circular braiding model and Heieck et al. [13] researched the influence of cover factor and Tobias Wehrkamp-Richter et al. [14] 
studied damage and failure of composites and E.E. Swery [15] has predicted the manufacturing of composite parts and experimented through the industrial robot.

However, studies on non-linear dynamic feature of a multiple-stage closed-loop gear transmission system for 3D circular braiding machine are scarce. Gear transmission is widely used in various fields. Many scholars have always focus on the research of gear system since 1990. The main research direction of gear system is the study of dynamic model and dynamic characteristics. There are many dynamic modeling methods. Kahraman, Singh [16] established a non-linear dynamic model of single-stage gear system considering error and backlash in 1990. Later, Kahraman, Singh [17] established a non-linear dynamic model of 3DOF gear system considering multiple incentive factors. Song [18] established a single-stage gear dynamic model considering friction and time-varying meshing stiffness. Liu [19] established a multiple-stage gear dynamic model considering other incentive factors. Cui [20] established a gear rotor dynamic model considering different incentive factors. Baguet [21] established a gear-rotor-bearing coupling dynamic model considering multiple incentive factors. $\mathrm{Li}$ [22] established a friction dynamic model considering multiple incentive factors. WEI Jing [23] established a multiple-degree-of-freedom gear dynamic model for high-speed locomotive considering bearing clearance, backlash, time-varying meshing stiffness. ZHANG Hui-bo [24] established a gear rotor dynamic model considering multiple incentive factors. According to above literature, research on gears has developed from single-stage single-degree-of-freedom gear system to multiple-stage multiple-degree-of-freedom gear system. Meanwhile, the solving methods of gear system include Floquet-Lyapunov method, harmonic balance method, average method, multiple-scale method and numerical method.

To the authors' knowledge, there is no research on non-linear dynamic characteristics of closed-loop gear transmission system for 3D circular braiding machine. The stability of the closed-loop gear transmission system with symmetrical structure in 3D braiding machine has a great influence on braiding quality, so the investigation of non-linear dynamic characteristics of the multiple-stage closed-loop gear transmission system with symmetrical structure for 3D circular braiding machine is necessary. This paper proposes Runge-Kutta method for solving the dynamic equations of the multiple-stage closed-loop gear transmission system with symmetrical structure for 3D circular braiding machine to help engineers effectively understand the dynamic displacement error and dynamic meshing force under different backlash and the random disturbance of meshing damping, which can provide a reference for realizing the smoothness of the closed-loop gear transmission system with symmetrical structure for 3D braiding machine, and have great practical significance for improving the braiding quality.

The remainder of this paper is organized as follows: Section 2 presents the outline of torsional vibration model of n-elements closed-loop gear transmission system and non-linear differential equations of torsional vibration are listed according to the outline of torsional vibration model. In Section 3, non-linear differential equations of torsional vibration are simulated by Matlab. Dynamic transmission error of the first pair of gears and dynamic meshing force of the first pair of gears are analyzed. Finally, Section 4 gives some brief conclusions.

\section{Torsional Vibration Model of n-Elements Closed-loop Gear Transmission System}

\subsection{Torsional Vibration Model of Gear System}

The outline of radial braiding machine is shown in Figure 1, and its transmission system is shown in Figure 2, which can be simplified to the n-elements closed-loop gear transmission system as shown in Figure 3. Assuming that the pure torsional vibration of gear pair in a radial braiding machine is considered, motors drive load to rotate through n-elements closed-loop gear transmission system. In order to establish and solve differential equations of n-elements gear transmission system in a radial braiding machine, rotational inertia of the shaft is distributed to the gear or rotor of each shaft by using the principle of functional equivalence and centroid invariance. The rotational inertia of the gear 
and rotor includes the equivalent mass of the shaft, and the equivalent dynamic model of n-elements closed-loop gear transmission system is shown in Figure 4.

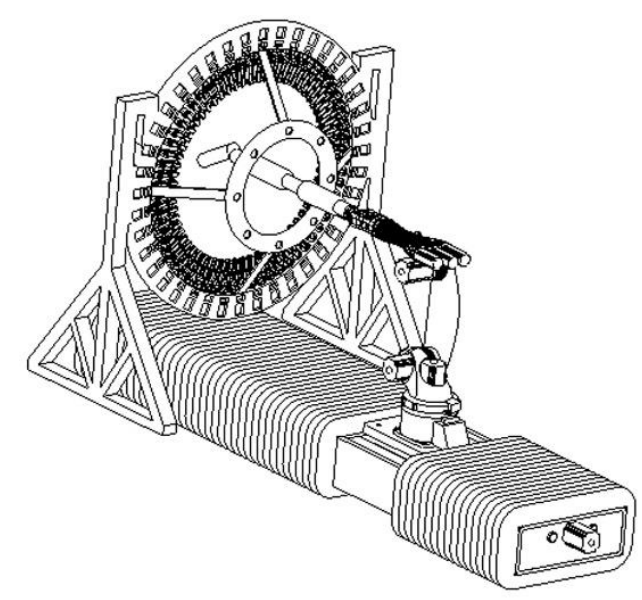

Figure 1. The outline of radial braiding machine.

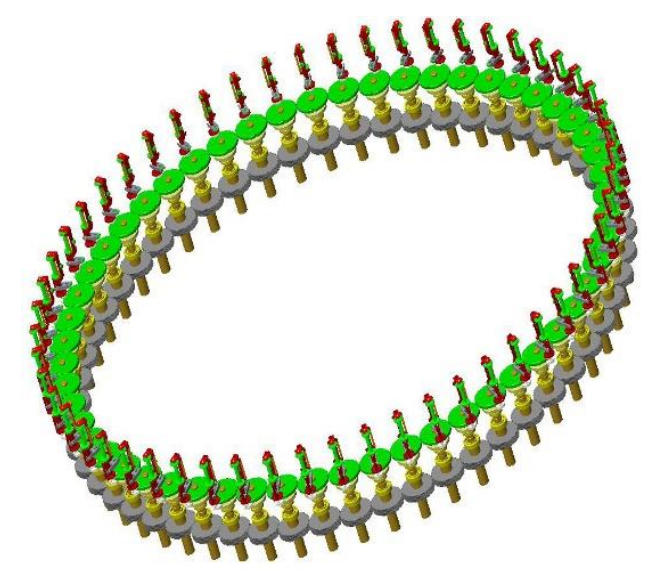

Figure 2. The actual outline of gear transmission system.

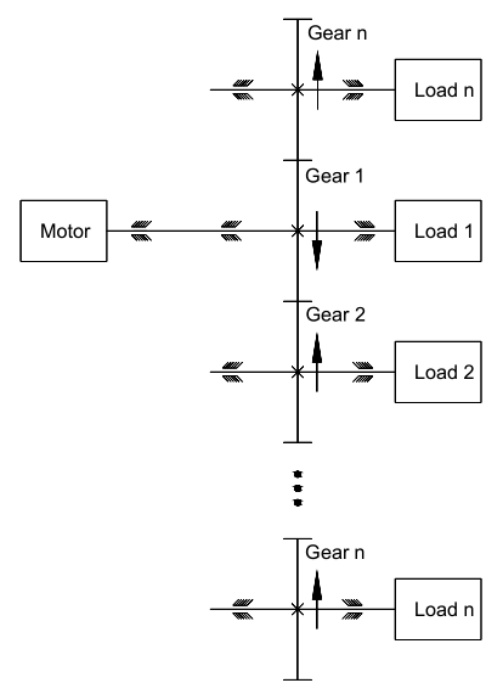

Figure 3. The outline of n-elements closed-loop gear transmission system. 


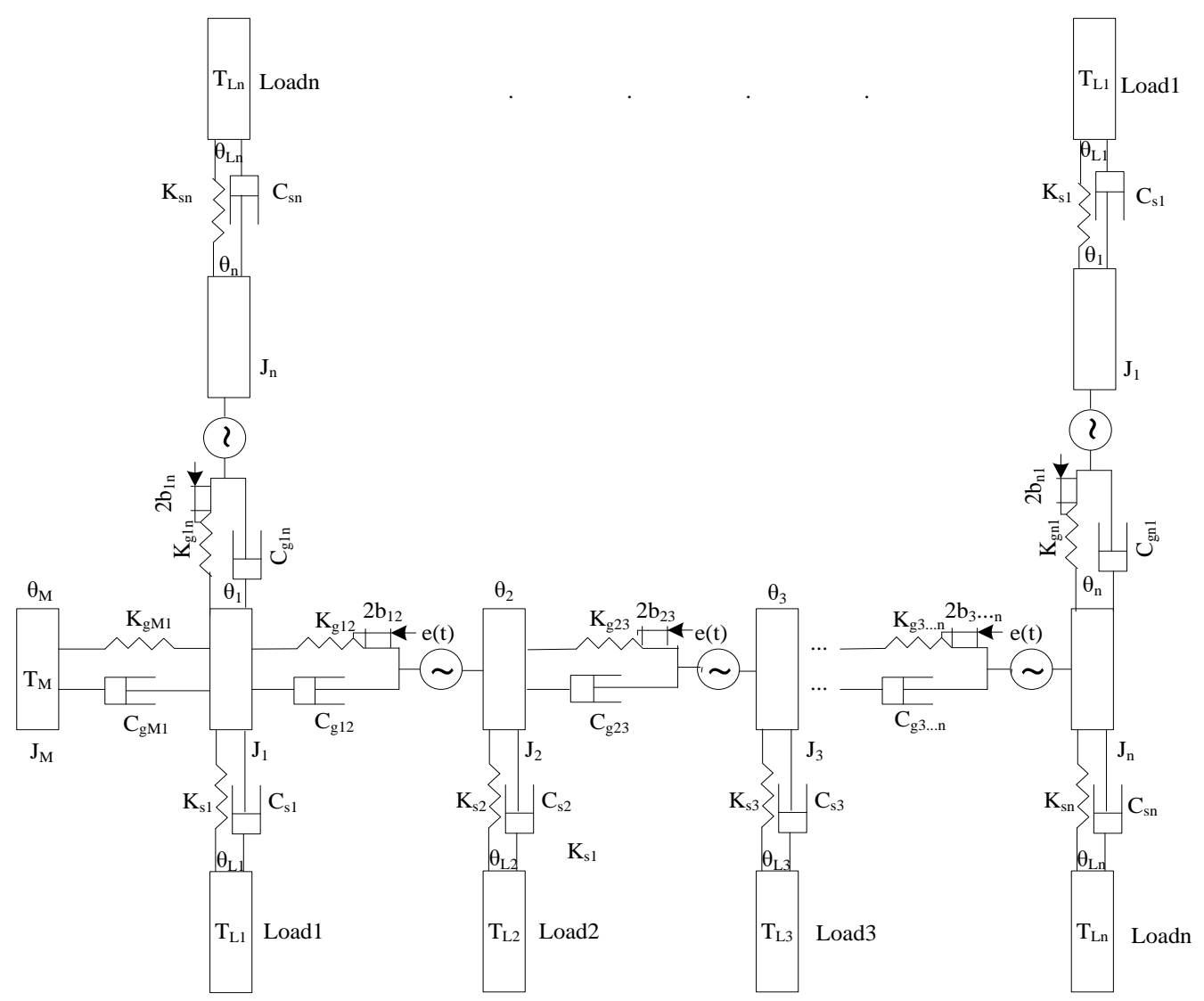

Figure 4. The equivalent dynamic model of n-elements closed-loop gear transmission system.

As shown in Figure $4, \theta_{M}$ is the angular displacement of motor, $\theta_{1}, \theta_{2} \ldots \theta_{n}$ are the angular displacements of gears, $\theta_{L 1}, \theta_{L 2} \ldots \theta_{L n}$ are the angular displacements of loads, $J_{M}$ is the rotational inertia of motor, $J_{1}, J_{2} \ldots J_{n}$ are the rotational inertia of gears, $J_{L 1}, J_{L 2} \ldots J_{L n}$ are the rotational inertia of loads, $C_{g M 1}$ is torsional damping of motor shaft, $C_{s 1}, C_{s 2} \ldots C_{s n}$ are torsional damping of output shafts, $K_{g M 1}$ is torsional rigidity of motor shaft, $K_{s 1}, K_{s 2} \ldots K_{s n}$ are torsional rigidity of output shafts, $K_{g 12}, K_{g 23} \ldots K_{g n(n+1)}$ are time-varying meshing stiffness between gear pairs, $C_{g 12}, C_{g 23} \ldots C_{g n(n+1)}$ are meshing damping, $b_{12}, b_{23} \ldots b_{n(n+1)}$ are half clearance of meshing teeth pairs, $T_{M}$ is the torque of motor, $T_{L 1}, T_{L 2} \ldots T_{L n}$ are torque of loads, $C_{g \Delta}$ is the random disturbance of meshing damping between gear pairs.

\subsection{Non-linear Differential Equations of Torsional Vibration}

As shown in Figure 4, the dynamic differential equation of n-elements gear transmission system with transmission error, the random disturbance of meshing damping, the random disturbance of input torque and backlash is obtained according to gear system dynamics and the theory of mechanical vibration [25-31]. The dynamic differential equation of n-elements gear transmission system with 
transmission error, the random disturbance of meshing damping, the random disturbance of input torque and backlash obtained by analysis is shown as (1):

$$
\left\{\begin{array}{c}
J_{M} \cdot \ddot{\theta}_{M}+C_{g M 1} \cdot\left(\dot{\theta}_{M}-\dot{\theta}_{1}\right)+K_{g M 1} \cdot\left(\theta_{M}-\theta_{1}\right)=T_{M} \\
J_{1} \cdot \ddot{\theta}_{1}+C_{g M 1} \cdot\left(\dot{\theta}_{1}-\dot{\theta}_{M}\right)+K_{g M 1} \cdot\left(\theta_{1}-\theta_{M}\right)+R_{b 1} \cdot W_{d 12}-R_{b 1} \cdot W_{d n 1}+C_{s 1} \cdot\left(\dot{\theta}_{1}-\dot{\theta}_{L 1}\right)+K_{s 1} \cdot\left(\theta_{1}-\theta_{L 1}\right)=0 \\
J_{L 1} \cdot \ddot{\theta}_{L 1}+C_{s 1} \cdot\left(\dot{\theta}_{L 1}-\dot{\theta}_{1}\right)+K_{s 1} \cdot\left(\theta_{L 1}-\theta_{1}\right)=-T_{L 1} \\
J_{2} \cdot \ddot{\theta}_{2}-R_{b 2} \cdot W_{d 12}+R_{b 2} \cdot W_{d 23}+C_{s 2} \cdot\left(\dot{\theta}_{2}-\dot{\theta}_{L 2}\right)+K_{s 2} \cdot\left(\theta_{2}-\theta_{L 2}\right)=0 \\
J_{L 2} \cdot \ddot{\theta}_{L 2}+C_{s 2} \cdot\left(\dot{\theta}_{L 2}-\dot{\theta}_{2}\right)+K_{s 2} \cdot\left(\theta_{L 2}-\theta_{2}\right)=-T_{L 2} \\
\cdot \\
\cdot \\
\cdot \\
J_{n} \cdot \ddot{\theta}_{n}-R_{b n} \cdot W_{d(n-1) n}+R_{b n} \cdot W_{d n 1}+C_{s n} \cdot\left(\dot{\theta}_{n}-\dot{\theta}_{L n}\right)+K_{s n} \cdot\left(\theta_{n}-\theta_{L n}\right)=0 \\
J_{L n} \cdot \ddot{\theta}_{L n}+C_{s n} \cdot\left(\dot{\theta}_{L n}-\dot{\theta}_{n}\right)+K_{s n} \cdot\left(\theta_{L n}-\theta_{n}\right)=-T_{L n}
\end{array}\right.
$$

where,

$$
\begin{aligned}
& W_{d 12}=\left(C_{\mathrm{g} 12}+C_{g \Delta}\right) \cdot\left[R_{b 1} \cdot \theta_{1}-R_{b 2} \cdot \theta_{2}-e(\mathrm{t})\right]+\mathrm{K}_{\mathrm{g} 12} \cdot g\left(\chi_{1}\right) \\
& W_{d 23}=\left(C_{\mathrm{g} 23}+C_{g \Delta}\right) \cdot\left[R_{b 2} \cdot \theta_{2}-R_{b 3} \cdot \theta_{3}-e(\mathrm{t})\right]+\mathrm{K}_{\mathrm{g} 23} \cdot g\left(\chi_{2}\right) \\
& W_{d \mathrm{nl}}=\left(C_{\mathrm{g} n 1}+C_{g \Delta}\right) \cdot\left[R_{b n} \cdot \theta_{n}-R_{b 1} \cdot \theta_{1}-e(\mathrm{t})\right]+\mathrm{K}_{\mathrm{g} n 1} \cdot g\left(\chi_{n}\right) \\
& \chi_{1}(\mathrm{t})=\left(R_{b 1} \cdot \theta_{1}-R_{b 2} \cdot \theta_{2}\right) \\
& \chi_{2}(\mathrm{t})=\left(R_{b 2} \cdot \theta_{2}-R_{b 3} \cdot \theta_{3}\right) \\
& \chi_{\mathrm{n}}(\mathrm{t})=\left(R_{b n} \cdot \theta_{n}-R_{b 1} \cdot \theta_{1}\right) \\
& \mathrm{g}\left(x_{1}\right)= \begin{cases}x_{1}(\mathrm{t})-\mathrm{e}(\mathrm{t})-\left(\bar{b}_{12}+\bar{b}_{\Delta}\right) & x_{1}(\mathrm{t})-\mathrm{e}(\mathrm{t})>\bar{b}_{12}+\bar{b}_{\Delta} \\
0 & \left|x_{1}(\mathrm{t})-\mathrm{e}(\mathrm{t})\right| \leq \bar{b}_{12}+\bar{b}_{\Delta} \\
x_{1}(\mathrm{t})-\mathrm{e}(\mathrm{t})+\left(\bar{b}_{12}+\bar{b}_{\Delta}\right) & x_{1}(\mathrm{t})-\mathrm{e}(\mathrm{t})<-\bar{b}_{12}-\bar{b}_{\Delta}\end{cases} \\
& \mathrm{g}\left(x_{2}\right)= \begin{cases}x_{2}(\mathrm{t})-\mathrm{e}(\mathrm{t})-\left(\bar{b}_{23}+\bar{b}_{\Delta}\right) & x_{2}(\mathrm{t})-\mathrm{e}(\mathrm{t})>\bar{b}_{23}+\bar{b}_{\Delta} \\
0 & \left|x_{2}(\mathrm{t})-\mathrm{e}(\mathrm{t})\right| \leq \bar{b}_{23}+\bar{b}_{\Delta} \\
x_{2}(\mathrm{t})-\mathrm{e}(\mathrm{t})+\left(\bar{b}_{23}+\bar{b}_{\Delta}\right) & x_{2}(\mathrm{t})-\mathrm{e}(\mathrm{t})<-\bar{b}_{23}-\bar{b}_{\Delta}\end{cases} \\
& \mathrm{g}\left(x_{n}\right)= \begin{cases}x_{\mathrm{n}}(\mathrm{t})-\mathrm{e}(\mathrm{t})-\left(\bar{b}_{n 1}+\bar{b}_{\Delta}\right) & x_{\mathrm{n}}(\mathrm{t})-\mathrm{e}(\mathrm{t})>\bar{b}_{n 1}+\bar{b}_{\Delta} \\
0 & \left|x_{n}(\mathrm{t})-\mathrm{e}(\mathrm{t})\right| \leq \bar{b}_{n 1}+\bar{b}_{\Delta} \\
x_{n}(\mathrm{t})-\mathrm{e}(\mathrm{t})+\left(\bar{b}_{n 1}+\bar{b}_{\Delta}\right) & x_{n}(\mathrm{t})-\mathrm{e}(\mathrm{t})<-\bar{b}_{n 1}-\bar{b}_{\Delta}\end{cases}
\end{aligned}
$$

where, $e(t)$ is the comprehensive transmission error of gear meshing, $\bar{b}_{12}, \bar{b}_{23} \ldots \bar{b}_{n 1}$ are dimensionless clearance, $W_{d 12} \ldots W_{d n 1}$ is the dynamic meshing force of gear, $x_{1}(t) \ldots x_{n}(t)$ is the dynamic transmission error, $\bar{b}_{12}=b_{12} / b_{e}, \bar{b}_{23}=b_{23} / b_{e} \ldots \bar{b}_{n 1}=b_{n 1} / b_{e}, b_{e}$ is the nominal size, $\bar{b}_{\Delta}$ is the random disturbance of dimensionless gear side clearance. For convenience of calculation, suppose $n=3$ is analyzed.

Because gear transmission system is the most important transmission form of mechanical equipment, which is widely used in various fields. However, the excessive dynamic displacement caused by the vibration response of the transmission system will affect the normal operation of the mechanical equipment. Therefore, in order to obtain the dimensionless dynamic displacement error of the gear pair, taking $q_{2}=\frac{R b_{1} \theta_{1}-R b_{2} \theta_{2}-e(t)}{b_{e}}$, the rest $q_{1} \ldots q_{7}$ also refers to the transmission error, and the others are similar. $m_{1}, m_{2}, m_{3}$ is the mass of gear. In order to obtain the dimensionless Equations (2a)-(2g), $m_{e 1}, m_{e 2}, m_{e 3}$ is the equivalent mass of gear. $J_{e 1}, J_{e 2}, J_{e 3}$ is the equivalent rotational inertia of gear. The others are similar.

In order to obtain the dimensionless dynamic displacement error of the gear pair, the dimensionless Equations (2a)-(2g) are obtained by adding, subtracting, multiplying and dividing the equations 
in (1). And taking $q_{1}=\theta_{M}-\theta_{1}, q_{2}=\frac{R_{b 1} \theta_{1}-R_{b 2} \theta_{2}-e(t)}{b_{e}}, q_{3}=\frac{R_{b 2} \theta_{2}-R_{b 3} \theta_{3}-e(t)}{b_{e}}, q_{4}=\frac{R_{b 3} \theta_{3}-R_{b 1} \theta_{1}-e(t)}{b_{e}}$, $q_{5}=\theta_{1}-\theta_{L 1}, q_{6}=\theta_{2}-\theta_{L 2}, q_{7}=\theta_{3}-\theta_{L 3}, m_{1}=\frac{J_{1}}{R_{b 1}{ }^{2}}, m_{2}=\frac{J_{2}}{R_{b 2}{ }^{2}}, m_{3}=\frac{J_{3}}{R_{b 3}{ }^{2}}, m_{e 1}=\frac{m_{1} m_{2}}{m_{1}+m_{2}}$, $m_{e 2}=\frac{m_{2} m_{3}}{m_{2}+m_{3}}, m_{e 3}=\frac{m_{1} m_{3}}{m_{1}+m_{3}}, \ddot{e}(t)=-e \cdot \omega^{2} \cdot \sin (\omega t), \Omega=\frac{\omega}{\omega_{n}}, J_{e 1}=\frac{J_{1} J_{M}}{J_{1}+J_{M}}, J_{e 2}=\frac{J_{1} J_{L 1}}{J_{1}+J_{L 1}}, J_{e 3}=\frac{J_{2} J_{L 2}}{J_{2}+J_{L 2}}$, $J_{e 4}=\frac{J_{3} I_{L 3}}{J_{3}+J_{L 3}}, \tau=w_{n} t$. The final dimensionless Equations (2a)-(2g) are as follows:

$$
\begin{aligned}
& \ddot{q}_{1}+\frac{C_{g M 1}}{J_{e 1} \cdot \omega_{n}} \cdot \dot{q}_{1}+\frac{K_{g M 1}}{J_{e 1} \cdot \omega_{n}^{2}} \cdot q_{1}-\frac{\left(C_{g 12}+C_{g \Delta}\right) \cdot R_{b 1} \cdot b_{e}}{J_{1} \cdot \omega_{n}} \cdot \dot{q}_{2}-\ldots \\
& \frac{K_{g 12} \cdot R_{b 1} \cdot b_{e}}{J_{1} \cdot \omega_{n}{ }^{2}} \cdot g\left(q_{2}\right)+\frac{\left(C_{g 31}+C_{g \Delta}\right) \cdot R_{b 1} \cdot b_{e}}{J_{1} \cdot \omega_{n}} \cdot \dot{q}_{4}+\frac{K_{g 31} \cdot b_{e} \cdot R_{b 1}}{J_{1} \omega_{n}{ }^{2}} \cdot g\left(q_{4}\right)-\ldots \\
& \frac{C_{s 1} \omega_{1}}{J_{1} \omega_{n}} \cdot \dot{q}_{5}-\frac{K_{s 1}}{J_{1} \omega_{n}^{2}} q_{5}=\frac{T_{M}}{J_{M} \omega_{n}^{2}} \\
& \ddot{q}_{2}+\frac{\left(C_{g 12}+C_{g \Delta}\right)}{m_{e 1} \cdot \omega_{n}} \cdot \dot{q}_{2}+\frac{K_{g 12}}{m_{e 1} \cdot \omega_{n}^{2}} \cdot g\left(q_{2}\right)-\frac{C_{g M 1}}{m_{1} \cdot R_{b 1} \cdot b_{e} \cdot w_{n}} \cdot \dot{q}_{1}-\ldots \\
& \frac{K_{g M 1}}{m_{1} \cdot R_{b 1} \cdot b_{e} \cdot \omega_{n}{ }^{2}} \cdot q_{1}-\frac{\left(C_{g 23}+C_{g \Delta}\right)}{m_{2} \cdot \omega_{n}} \cdot \dot{q}_{3}-\frac{K_{g 23}}{m_{2} \cdot \omega_{n}{ }^{2}} \cdot g\left(q_{3}\right)-\ldots \\
& \frac{\left(C_{g 31}+C_{g \Delta}\right)}{m_{1} \cdot \omega_{n}} \cdot \dot{q}_{4}-\frac{K_{g 31}}{m_{1} \cdot \omega_{n}^{2}} \cdot g\left(q_{4}\right)+\frac{C_{s 1}}{m_{1} \cdot R_{b 1} \cdot b_{e} \cdot \omega_{n}} \cdot \dot{q}_{5}+\ldots \\
& \frac{K_{s 1}}{m_{1} \cdot R_{b 1} \cdot b_{e} \cdot \omega_{n}{ }^{2}} \cdot q_{5}-\frac{C_{s 2}}{m_{2} \cdot R_{b 2} \cdot b_{e} \cdot \omega_{n}} \cdot \dot{q}_{6}-\frac{K_{s 2}}{m_{2} \cdot R_{b 2} \cdot b_{e} \cdot \omega_{n}^{2}} \cdot q_{6} \ldots \\
& =\frac{e}{b_{e}} \cdot \Omega^{2} \cdot \sin (\Omega \cdot \tau) \\
& \ddot{q}_{3}+\frac{\left(C_{g 23}+C_{g \Delta}\right)}{m_{e 2} \cdot \omega_{n}} \cdot \dot{q}_{3}+\frac{K_{g 23}}{m_{e 2} \cdot \omega_{n}{ }^{2}} \cdot g\left(q_{3}\right)-\frac{\left(C_{g 12}+C_{g \Delta}\right)}{m_{2} \omega_{n}} \cdot \dot{q}_{2}-\ldots \\
& \frac{K_{g 12}}{m_{2} \cdot \omega_{n}^{2}} \cdot g\left(q_{2}\right)-\frac{\left(C_{g 31}+C_{g \Delta}\right)}{m_{3} \cdot \omega_{n}} \cdot \dot{q}_{4}-\frac{K_{g 31}}{m_{3} \cdot \omega_{n}^{2}} \cdot g\left(q_{4}\right)+\frac{C_{s 2}}{b_{e} \cdot R_{b 2} \cdot m_{2} \cdot \omega_{n}} \cdot \dot{q}_{6}+\ldots \\
& \frac{K_{s 2}}{b_{e} \cdot R_{b 2} \cdot m_{2} \cdot \omega_{n}{ }^{2}} \cdot q_{6}-\frac{C_{s 3}}{b_{e} \cdot R_{b 3} \cdot m_{3} \cdot \omega_{n}} \cdot \dot{q}_{7}-\frac{K_{s 3}}{b_{e} \cdot R_{b 3} \cdot m_{3} \cdot \omega_{n}{ }^{2}} \cdot q_{7} \ldots \\
& =\frac{e}{b_{e}} \cdot \Omega^{2} \cdot \sin (\Omega \cdot \tau) \\
& \ddot{q}_{4}+\frac{\left(C_{g 31}+C_{g \Delta}\right)}{m_{e 3} \cdot \omega_{n}} \cdot \dot{q}_{4}+\frac{K_{831}}{m_{e 3} \cdot \omega_{n}{ }^{2}} \cdot g\left(q_{4}\right)-\frac{\left(C_{g 23}+C_{g \Delta}\right)}{m_{3} \cdot \omega_{n}} \cdot \dot{q}_{3}-\frac{K_{g 23}}{m_{3} \cdot \omega_{n}{ }^{2}} \cdot g\left(q_{3}\right)+\ldots \\
& \frac{C_{g M 1}}{m_{1} \cdot R_{b 1} \cdot b_{e} \cdot \omega_{n}} \cdot \dot{q}_{1}+\frac{K_{g M 1}}{m_{1} \cdot R_{b 1} \cdot b_{e} \cdot \omega_{n}^{2}} \cdot q_{1}-\frac{\left(C_{g 12}+C_{g \Delta}\right)}{m_{1} \cdot \omega_{n}} \cdot \dot{q}_{2}-\ldots \\
& \frac{K_{g 12}}{m_{1} \cdot \omega_{n}^{2}} \cdot g\left(q_{2}\right)-\frac{C_{s 1}}{m_{1} \cdot R_{b 1} \cdot b_{b} \cdot \omega_{n}} \cdot \dot{q}_{5}-\frac{K_{s 1}}{m_{1} \cdot R_{b 1} \cdot b_{e} \cdot \omega_{n}^{2}} \cdot q_{5}+\ldots
\end{aligned}
$$

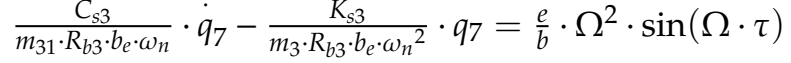

$$
\begin{aligned}
& \ddot{q}_{5}+\frac{C_{s 1}}{J_{e 2} \cdot \omega_{n}} \dot{q}_{5}+\frac{K_{s 1}}{J_{e 2} \cdot \omega_{n}^{2}} q_{5}-\frac{C_{g M 1}}{J_{1} \cdot \omega_{n}} \dot{q}_{1}-\frac{K_{g M 1}}{J_{1} \cdot \omega_{n}^{2}} q_{1}+\ldots \\
& \frac{R_{b 1} \cdot b_{e} \cdot\left(C_{g 12}+C_{g \Delta}\right)}{J_{1} \cdot \omega_{n}} \cdot q_{2}+\frac{R_{b 1} \cdot b_{e} \cdot K_{g 12}}{J_{1} \cdot \omega_{n}{ }^{2}} \cdot g\left(q_{2}\right)-\ldots \\
& \frac{R_{b 1} \cdot b_{b} \cdot\left(C_{g 31}+C_{g \Delta}\right)}{J_{1} \cdot \omega_{n}} \cdot q_{4}-\frac{R_{b 1} \cdot b_{e} \cdot K_{g 31}}{J_{1} \cdot \omega_{n}{ }^{2}} \cdot g\left(q_{4}\right)=\frac{T_{L 1}}{J_{L 1} \cdot \omega_{n}{ }^{2}} \\
& \ddot{q}_{6}+\frac{C_{s 2}}{J_{e 3} \cdot \omega_{n}} \cdot \dot{q}_{6}+\frac{K_{s 2}}{J_{e 3} \cdot \omega_{n}^{2}} \cdot q_{6}-\frac{\left(C_{g 12}+C_{g \Delta}\right) \cdot R_{b 2} \cdot b_{e}}{J_{2} \cdot \omega_{n}} \cdot \dot{q}_{2}-\ldots \\
& \frac{K_{g 12} \cdot R_{b 2} \cdot b_{e}}{J_{2} \cdot \omega_{n}{ }^{2}} \cdot g\left(q_{2}\right)+\frac{\left(C_{g 23}+C_{g \Delta}\right) \cdot R_{b 2} \cdot b_{e}}{J_{2} \cdot \omega_{n}} \cdot \dot{q}_{3}+\frac{K_{g 23} \cdot R_{b 2} \cdot b_{e}}{J_{2} \cdot \omega_{n}{ }^{2}} \cdot g\left(q_{3}\right) \ldots \\
& =\frac{T_{L 2}}{J_{L 2} \cdot \omega_{n}^{2}} \\
& \ddot{q}_{7}+\frac{C_{s 3}}{J_{e 4} \cdot \omega_{n}} \cdot \dot{q}_{7}+\frac{K_{s 3}}{J_{e 4} \cdot \omega_{n}{ }^{2}} \cdot q_{7}-\frac{\left(C_{g 23}+C_{g \Delta}\right) \cdot R_{b 3} \cdot b_{e}}{J_{3} \cdot \omega_{n}} \cdot \dot{q}_{3}-\ldots \\
& \frac{K_{g 23} \cdot R_{b 3} \cdot b_{e}}{J_{3} \cdot \omega_{n_{n}}{ }^{2}} \cdot g\left(q_{3}\right)+\frac{\left(C_{g 31}+C_{g \Delta}\right) \cdot R_{b 3} \cdot b_{e}}{J_{3} \cdot \omega_{n}} \cdot \dot{q}_{4}+\frac{K_{g 31} \cdot R_{b 3} \cdot b_{e}}{J_{3} \cdot \omega_{n}{ }^{2}} \cdot g\left(q_{4}\right) \ldots \\
& =\frac{T_{L 3}}{J_{L 3} \cdot \omega_{n}{ }^{2}}
\end{aligned}
$$

In order to obtain simplified dimensionless Equations (3a)-(3g), substituting

$$
\xi_{11}=\frac{C_{g M 1}}{2 \cdot J_{e 1} \cdot \omega_{n}}, k_{11}=\frac{K_{g M 1}}{J_{e 1} \cdot \omega_{n}^{2}{ }^{2}}, \xi_{12}=\frac{\left(C_{g 12}+C_{g \Delta}\right) \cdot R_{b 1} \cdot b_{e}}{2 \cdot J_{1} \cdot \omega_{n}}, k_{12}=\frac{K_{g 12} \cdot R_{b 1} \cdot b_{e}}{J_{1} \cdot \omega_{n}^{2}}
$$




$$
\begin{aligned}
& \xi_{13}=\frac{\left(C_{g 31}+C_{g \Delta}\right) \cdot R_{b 1} \cdot b_{e}}{2 \cdot J_{1} \cdot \omega_{n}}, k_{13}=\frac{K_{g 31} \cdot R_{b 1} \cdot b_{e}}{J_{1} \cdot \omega_{n}^{2}}, \xi_{14}=\frac{C_{s 1}}{2 \cdot J_{1} \cdot \omega_{n}}, k_{14}=\frac{K_{s 1}}{J_{1} \cdot \omega_{n}{ }^{2}}, \\
& f_{1}=\frac{T_{M}}{J_{M} \cdot \omega_{n}^{2}}, \xi_{21}=\frac{C_{s 1}}{2 \cdot J_{e 2} \cdot \omega_{n}}, k_{21}=\frac{K_{s 1}}{J_{e 2} \cdot \omega_{n}{ }^{2}}, \xi_{22}=\frac{C_{g M 1}}{2 \cdot J_{1} \cdot \omega_{n}}, k_{22}=\frac{K_{g M 1}}{J_{1} \cdot \omega_{n}{ }^{2}}, \\
& \xi_{23}=\frac{\left(C_{g 12}+C_{g \Delta}\right) \cdot R_{b 1} \cdot b_{e}}{2 \cdot J_{1} \cdot \omega_{n}}, k_{23}=\frac{K_{g 12} \cdot R_{b 1} \cdot b_{e}}{J_{1} \cdot \omega_{n}^{2}}, \xi_{24}=\frac{\left(C_{g 31}+C_{g \Delta}\right) \cdot R_{b 1} \cdot b_{e}}{2 \cdot J_{1} \cdot \omega_{n}}, \\
& k_{24}=\frac{K_{g 31} \cdot R_{b 1} \cdot b_{e}}{J_{1} \cdot \omega_{n}^{2}}, f_{2}=\frac{T_{L 1}}{J_{L 1} \cdot \omega_{n}^{2}}, \xi_{31}=\frac{C_{s 2}}{2 \cdot J_{e 3} \cdot \omega_{n}}, k_{31}=\frac{K_{s 2}}{J_{e 3} \cdot \omega_{n}^{2}}, \\
& \xi_{32}=\frac{\left(C_{g 12}+C_{g \Delta}\right) \cdot R_{b 2} \cdot b_{e}}{2 \cdot J_{2} \cdot \omega_{n}}, k_{32}=\frac{K_{g 12} \cdot R_{b 2} \cdot b_{e}}{J_{2} \cdot \omega_{n}^{2}}, \xi_{33}=\frac{\left(C_{g 23}+C_{g \Delta}\right) \cdot R_{b 2} \cdot b_{e}}{2 \cdot J_{2} \cdot \omega_{n}}, \\
& k_{33}=\frac{K_{g 23} \cdot R_{b 2} \cdot b_{e}}{J_{2} \cdot \omega_{n}^{2}}, f_{3}=\frac{T_{L 2}}{J_{L 2} \cdot \omega_{n}^{2}}, \xi_{41}=\frac{C_{s 3}}{2 \cdot J_{e 4} \cdot \omega_{n}}, k_{41}=\frac{K_{s 3}}{J_{e 4} \cdot \omega_{n}^{2}}, \\
& \xi_{42}=\frac{\left(C_{g 23}+C_{g \Delta}\right) \cdot R_{b 3} \cdot b_{e}}{2 \cdot J_{3} \cdot \omega_{n}}, k_{42}=\frac{K_{g 23} \cdot R_{b 3} \cdot b_{e}}{J_{3} \cdot \omega_{n}^{2}}, \xi_{43}=\frac{\left(C_{g 31}+C_{g \Delta}\right) \cdot R_{b 3} \cdot b_{e}}{2 \cdot J_{3} \cdot \omega_{n}}, \\
& k_{43}=\frac{K_{g 31} \cdot R_{b 3} \cdot b_{e}}{J_{3} \cdot \omega_{n}^{2}}, f_{4}=\frac{T_{L 3}}{J_{L 3} \cdot \omega_{n}^{2}}, \xi_{51}=\frac{\left(C_{g 12}+C_{g \Delta}\right)}{2 \cdot m_{e 1} \cdot \omega_{n}}, k_{51}=\frac{K_{g 12}}{m_{e 1} \cdot \omega_{n}{ }^{2}}, \\
& \xi_{52}=\frac{C_{g M 1}}{2 \cdot m_{1} \cdot R_{b 1} \cdot b_{e} \cdot \omega_{n}}, k_{52}=\frac{K_{g M 1}}{m_{1} \cdot R_{b 1} \cdot b_{e} \cdot \omega_{n}{ }^{2}}, \xi_{53}=\frac{\left(C_{g 23}+C_{g \Delta}\right)}{2 \cdot m_{2} \cdot \omega_{n}}, k_{53}=\frac{K_{g 23}}{m_{2} \cdot \omega_{n}{ }^{2}}, \\
& \xi_{54}=\frac{\left(C_{g 31}+C_{g \Delta}\right)}{2 \cdot m_{1} \cdot \omega_{n}}, k_{54}=\frac{K_{g 31}}{m_{1} \cdot \omega_{n}{ }^{2}}, \xi_{55}=\frac{C_{s 1}}{2 \cdot R_{b 1} \cdot m_{1} \cdot b_{e} \cdot \omega_{n}}, k_{55}=\frac{K_{s 1}}{R_{b 1} \cdot m_{1} \cdot b_{e} \cdot \omega_{n}{ }^{2}}, \\
& \xi_{56}=\frac{C_{s 2}}{2 \cdot R_{b 2} \cdot m_{2} \cdot b_{e} \cdot \omega_{n}}, k_{56}=\frac{K_{s 2}}{R_{b 2} \cdot m_{2} \cdot b_{e} \cdot \omega_{n}^{2}}, f_{5}=\frac{e}{b} \cdot \Omega^{2} \cdot \sin (\Omega \cdot \tau), \\
& \xi_{61}=\frac{\left(C_{g 23}+C_{g \Delta}\right)}{2 \cdot m_{e 2} \cdot \omega_{n}}, k_{61}=\frac{K_{g 23}}{m_{e 2} \cdot \omega_{n}{ }^{2}}, \xi_{62}=\frac{\left(C_{g 12}+C_{g \Delta}\right)}{2 \cdot m_{2} \cdot \omega_{n}}, k_{62}=\frac{K_{g 12}}{m_{2} \cdot \omega_{n}{ }^{2}} \\
& \xi_{63}=\frac{\left(C_{g 31}+C_{g \Delta}\right)}{2 \cdot m_{3} \cdot \omega_{n}}, k_{63}=\frac{K_{g 31}}{m_{3} \cdot \omega_{n}{ }^{2}}, \xi_{64}=\frac{C_{s 2}}{2 \cdot m_{2} \cdot R_{2} \cdot b_{e} \cdot \omega_{n}}, k_{64}=\frac{K_{s 2}}{m_{2} \cdot R_{2} \cdot b_{e} \cdot \omega_{n}{ }^{2}}, \\
& \xi_{65}=\frac{C_{s 3}}{2 \cdot m_{3} \cdot R_{b 3} \cdot b_{e} \cdot \omega_{n}}, k_{65}=\frac{K_{s 3}}{m_{3} \cdot R_{b 3} \cdot b_{e} \cdot \omega_{n}^{2}}, f_{6}=\frac{e}{b} \cdot \Omega^{2} \cdot \sin (\Omega \cdot \tau), \\
& \xi_{71}=\frac{\left(C_{g 31}+C_{g \Delta}\right)}{2 \cdot m_{e 3} \cdot \omega_{n}}, k_{71}=\frac{K_{g 31}}{m_{e 3} \cdot \omega_{n}{ }^{2}}, \xi_{72}=\frac{\left(C_{g 23}+C_{g \Delta}\right)}{2 \cdot m_{3} \cdot \omega_{n}}, k_{72}=\frac{K_{g 23}}{m_{3} \cdot \omega_{n}{ }^{2}}, \\
& \xi_{73}=\frac{C_{g M 1}}{2 \cdot m_{1} \cdot R_{b 1} \cdot b_{e} \cdot \omega_{n}}, k_{73}=\frac{K_{g M 1}}{m_{1} \cdot R_{b 1} \cdot b_{e} \cdot \omega_{n}{ }^{2}}, \xi_{74}=\frac{\left(C_{g 12}+C_{g \Delta}\right)}{2 \cdot m_{1} \cdot \omega_{n}}, k_{74}=\frac{K_{g 12}}{m_{1} \cdot \omega_{n}{ }^{2}}, \\
& \xi_{75}=\frac{C_{s 1}}{2 \cdot R_{b 1} \cdot m_{1} \cdot b_{e} \cdot \omega_{n}}, k_{75}=\frac{K_{s 2}}{R_{b 1} \cdot m_{1} \cdot b_{e} \cdot \omega_{n}^{2}}, \xi_{76}=\frac{C_{s 3}}{2 \cdot m_{3} \cdot R_{b 3} \cdot b_{e} \cdot \omega_{n}}, \\
& k_{76}=\frac{K_{s 3}}{m_{3} \cdot R_{b 3} \cdot b_{e} \cdot \omega_{n}^{2}}, f_{7}=\frac{e}{b} \cdot \Omega^{2} \cdot \sin (\Omega \cdot \tau)
\end{aligned}
$$

into (2a)-(2g). 
The final dimensionless equation obtained by simplifying the above formula is as follows:

$$
\begin{gathered}
\ddot{q}_{1}+2 \cdot \xi_{11} \cdot \dot{q}_{1}+k_{11} \cdot q_{1}-2 \cdot \xi_{12} \cdot \dot{q}_{2}-k_{12} \cdot g\left(q_{2}\right)+2 \cdot \xi_{13} \cdot \dot{q}_{4}+\ldots \\
k_{13} \cdot g\left(q_{4}\right)-2 \cdot \xi_{14} \cdot \dot{q}_{5}-k_{14} \cdot q_{5}=f_{1} \\
\ddot{q}_{2}+2 \cdot \xi_{51} \cdot \dot{q}_{2}+k_{51} \cdot g\left(q_{2}\right)-2 \cdot \xi_{52} \cdot \dot{q}_{1}-k_{52} \cdot q_{1}-2 \cdot \xi_{53} \cdot \dot{q}_{3}-k_{53} \cdot g\left(q_{3}\right)-\ldots \\
2 \cdot \xi_{54} \cdot \dot{q}_{4}-k_{54} \cdot g\left(q_{4}\right)+2 \cdot \xi_{55} \cdot \dot{q}_{5}+k_{55} \cdot q_{5}-2 \cdot \xi_{56} \cdot \dot{q}_{6}-k_{56} \cdot q_{6}=f_{5} \\
\ddot{q}_{3}+2 \cdot \xi_{61} \cdot \dot{q}_{3}+k_{61} \cdot g\left(q_{3}\right)-2 \cdot \xi_{62} \cdot \dot{q}_{2}-k_{62} \cdot g\left(q_{2}\right)-2 \cdot \xi_{63} \cdot \dot{q}_{4}-k_{63} \cdot g\left(q_{4}\right)+\ldots \\
2 \cdot \xi_{64} \cdot \dot{q}_{6}+k_{64} \cdot q_{6}-2 \cdot \xi_{65} \cdot \dot{q}_{7}-k_{65} \cdot q_{7}=f_{6} \\
\ddot{q}_{4}+2 \cdot \xi_{71} \cdot \dot{q}_{4}+k_{71} \cdot g\left(q_{4}\right)-2 \cdot \xi_{72} \cdot \dot{q}_{3}-k_{72} \cdot g\left(q_{3}\right)+2 \cdot \xi_{73} \cdot \dot{q}_{1}+k_{73} \cdot q_{1}-\ldots \\
-2 \cdot \xi_{74} \cdot \dot{q}_{2}-k_{74} \cdot g\left(q_{2}\right)-2 \cdot \xi_{75} \cdot \dot{q}_{5}-k_{75} \cdot q_{5}+2 \cdot \xi_{76} \cdot \dot{q}_{7}+k_{76} \cdot q_{7}=f_{7} \\
\quad \ddot{q}_{5}+2 \cdot \xi_{21} \cdot \dot{q}_{5}+k_{21} \cdot q_{5}-2 \cdot \xi_{22} \cdot \dot{q}_{1}-k_{22} \cdot q_{1}+2 \cdot \xi_{23} \cdot \dot{q}_{2}+\ldots \\
k_{23} \cdot g\left(q_{2}\right)-2 \cdot \xi_{24} \cdot \dot{q}_{4}-k_{24} \cdot g\left(q_{4}\right)=f_{2} \\
\ddot{q}_{6}+2 \cdot \xi_{31} \cdot \dot{q}_{6}+k_{31} \cdot q_{6}-2 \cdot \xi_{32} \cdot \dot{q}_{2}-k_{32} \cdot g\left(q_{2}\right)+2 \cdot \xi_{33} \cdot \dot{q}_{3}+k_{33} \cdot g\left(q_{3}\right)=f_{3} \\
\ddot{q}_{7}+2 \cdot \xi_{41} \cdot \dot{q}_{7}+k_{41} \cdot q_{7}-2 \cdot \xi_{42} \cdot \dot{q}_{3}-k_{42} \cdot g\left(q_{3}\right)+2 \cdot \xi_{43} \cdot \dot{q}_{4}+k_{43} \cdot g\left(q_{4}\right)=f_{4}
\end{gathered}
$$

In order to solve the above Equations (3a)-(3g) is transformed into the following equations:

$$
\begin{aligned}
& \begin{aligned}
\bar{Z} & =\left(\begin{array}{llllllllllllll}
z_{1} & z_{2} & z_{3} & z_{4} & z_{5} & z_{6} & z_{7} & z_{8} & z_{9} & z_{10} & z_{11} & z_{12} & z_{13} & z_{14}
\end{array}\right)^{T} \\
& =\left(\begin{array}{llllllllllllllll}
q_{1} & \dot{q}_{1} & q_{2} & \dot{q}_{2} & q_{3} & \dot{q}_{3} & q_{4} & \dot{q}_{4} & q_{5} & \dot{q}_{5} & q_{6} & \dot{q}_{6} & q_{7} & \dot{q}_{7}
\end{array}\right)^{T}
\end{aligned} \\
& \frac{d z_{1}}{d \tau}=z_{2} \\
& d z_{2}=\ddot{q}_{1}=f_{1}-2 \cdot \xi_{11} \cdot z_{2}-k_{11} \cdot z_{1}+2 \cdot \xi_{12} \cdot z_{4}+k_{12} \cdot g\left(z_{3}\right)-2 \cdot \xi_{13} \cdot z_{8}-\ldots \\
& k_{13} \cdot g\left(z_{7}\right)+2 \cdot \xi_{14} \cdot z_{10}+k_{14} \cdot z_{9} \\
& \frac{d z_{3}}{d \tau}=z_{4} \\
& d z_{4}=\ddot{q}_{2}=f_{5}-2 \cdot \xi_{51} \cdot z_{4}-k_{51} \cdot g\left(z_{3}\right)+2 \cdot \xi_{52} \cdot z_{2}+k_{52} \cdot z_{1}+2 \cdot \xi_{53} \cdot z_{6}+\ldots \\
& k_{53} \cdot g\left(z_{5}\right)+2 \cdot \xi_{54} \cdot z_{8}+k_{54} \cdot g\left(z_{7}\right)-2 \cdot \xi_{55} \cdot z_{10}-k_{55} \cdot z_{9}+2 \cdot \xi_{56} \cdot z_{12}+k_{56} \cdot z_{11} \\
& \frac{d z_{5}}{d \tau}=z_{6} \\
& d z_{6}=\ddot{q}_{3}=f_{6}-2 \cdot \xi_{61} \cdot z_{6}-k_{61} \cdot g\left(z_{5}\right)+2 \cdot \xi_{62} \cdot z_{4}+k_{62} \cdot g\left(z_{3}\right)+2 \cdot \xi_{63} \cdot z_{8}+\ldots \\
& k_{63} \cdot g\left(z_{7}\right)-2 \cdot \xi_{64} \cdot z_{12}-k_{64} \cdot z_{11}+2 \cdot \xi_{65} \cdot z_{14}+k_{65} \cdot z_{13} \\
& \frac{d z_{7}}{d \tau}=z_{8} \\
& d z_{8}=\ddot{q}_{4}=f_{7}-2 \cdot \xi_{71} \cdot z_{8}-k_{71} \cdot g\left(z_{7}\right)+2 \cdot \xi_{72} \cdot z_{6}+k_{72} \cdot g\left(z_{5}\right)-2 \cdot \xi_{73} \cdot z_{2}-\ldots \\
& k_{73} \cdot z_{1}+2 \cdot \xi_{74} \cdot z_{4}+k_{74} \cdot g\left(z_{3}\right)+2 \cdot \xi_{75} \cdot z_{10}+k_{75} \cdot z_{9}-2 \cdot \xi_{76} \cdot z_{14}-k_{76} \cdot z_{13} \\
& \frac{d z_{9}}{d \tau}=z_{10} \\
& d z_{10}=\ddot{q}_{5}=f_{2}-2 \cdot \xi_{21} \cdot z_{10}-k_{21} \cdot z_{9}+2 \cdot \xi_{22} \cdot z_{2}+k_{22} \cdot z_{1}-2 \cdot \xi_{23} \cdot z_{4}-\ldots \\
& k_{23} \cdot g\left(z_{3}\right)+2 \cdot \xi_{24} \cdot z_{8}+k_{24} \cdot g\left(z_{7}\right) \\
& \frac{d z_{11}}{d \tau}=z_{12}
\end{aligned}
$$




$$
\begin{aligned}
& d z_{12}=\ddot{q}_{6}=f_{3}-2 \cdot \xi_{31} \cdot z_{12}-k_{31} \cdot z_{11}+2 \cdot \xi_{32} \cdot z_{4}+k_{32} \cdot g\left(z_{3}\right)-\ldots \\
& 2 \cdot \xi_{33} \cdot z_{6}-k_{33} \cdot g\left(z_{5}\right) \\
& \frac{d z_{13}}{d \tau}=z_{14} \\
& d z_{14}=\ddot{q}_{7}=f_{4}-2 \cdot \xi_{41} \cdot z_{14}-k_{41} \cdot z_{13}+2 \cdot \xi_{42} \cdot z_{6}+k_{42} \cdot g\left(z_{5}\right)-\ldots \\
& 2 \cdot \xi_{43} \cdot z_{8}-k_{43} \cdot g\left(z_{7}\right)
\end{aligned}
$$

\section{The Simulation Analysis of MATLAB}

Taking gear transmission system of radial braiding machine shown in Figure 2 as an example, the dynamic differential equation of n-elements gear transmission system with static transmission error, the random disturbance of meshing damping and backlash are established. The basic parameters of gears are shown in Table 1. The rotational inertia of motor is $J_{M}=0.03 \mathrm{~kg} \cdot \mathrm{m}^{2}$, the speed is $n=1000 \mathrm{r} / \mathrm{min}$, the length of motor and gear shaft are $l_{1}=80 \mathrm{~mm}, l_{2}=50 \mathrm{~mm}$, the diameter is respectively $d_{1}=10 \mathrm{~mm}, d_{2}=20 \mathrm{~mm}$, half-tooth side clearance of tooth pairs is respectively $b_{12}=2.65 \times 10^{-6} \mathrm{~mm}, b_{12}=2.65 \times 10^{-5} \mathrm{~mm}, b_{12}=2.65 \times 10^{-4} \mathrm{~mm}$, motor and load torque is respectively $T_{M}=44 \mathrm{~N} \cdot \mathrm{m}, T_{L}=2 \mathrm{~N} \cdot \mathrm{m}$. Meanwhile, the dynamic differential equation of n-elements gear transmission system is simulated by Runge-Kutta method, and dynamic response results are obtained.

Table 1. The basic parameters of closed-loop gear transmission system.

\begin{tabular}{cccc}
\hline & Gear 1 & Gear 2 & Gear 3 \\
\hline Modulus/mm & 4 & 4 & 4 \\
Tooth number z & 30 & 30 & 30 \\
Tooth width B/mm & 10 & 10 & 10 \\
Pressure angle $\alpha /\left(^{\circ}\right)$ & 20 & 20 & 20 \\
Modification coefficient & 0.5047 & 0.5047 & 0.5047 \\
\hline
\end{tabular}

\subsection{The Analysis about Dynamic Transmission Error of the First Pair of Gears}

Time domain diagrams of dynamic transmission error of the first pair of gears at $b_{12}=2.65 \times 10^{-6} \mathrm{~m}, b_{12}=2.65 \times 10^{-5} \mathrm{~m}, b_{12}=2.65 \times 10^{-4} \mathrm{~m}$ are shown as Figures $5-7$, and the frequency domain diagram of dynamic transmission error of the first pair of gears at $b_{12}=2.65 \times 10^{-5} \mathrm{~m}$ is shown as Figure 8. From Figures 5-7, it is known that the dynamic transmission error will fluctuate with the increase of half-tooth side clearance of the first pair of gears, while the fluctuation trend of the other pair of gear is similar. From Figure 8, it can be seen that the amplitude of dynamic transmission error (DTE) is larger at $325 \mathrm{~Hz}, 675 \mathrm{~Hz}, 1000 \mathrm{~Hz}$ when $\mathrm{b}_{12}=2.65 \times 10^{-5} \mathrm{~m}, \mathrm{n}=1000 \mathrm{r} / \mathrm{min}$. Since a constant term of half-tooth side clearance, there is a large amplitude of dynamic transmission error (DTE) at $0 \mathrm{~Hz}$. Since the speed of the system has a great influence on the dynamic transmission error (DTE), and the dynamic transmission error (DTE) is a key factor affecting the stability of the system. RMS and the mean value of DTE under the different speed is shown as Figures 9 and 10. From Figures 9 and 10, the fluctuation trend of RMS and the mean value of DTE under the different speed is basically the same. RMS and the mean value of DTE are stable when the speed is less than $5000 \mathrm{r} / \mathrm{min}$. While RMS and the mean value of DTE are decreasing because of backlash, which can lead to a large proportion of gear separation and lateral contact when the speed is greater than $5000 \mathrm{r} / \mathrm{min}$. 


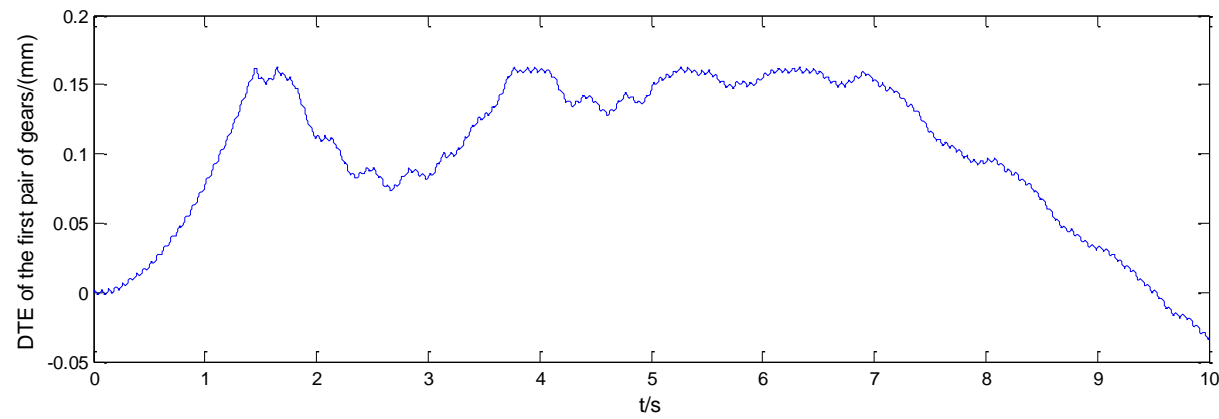

Figure 5. The dynamic transmission error of the first pair of gears at $b_{12}=2.65 \times 10^{-6} \mathrm{~m}$.

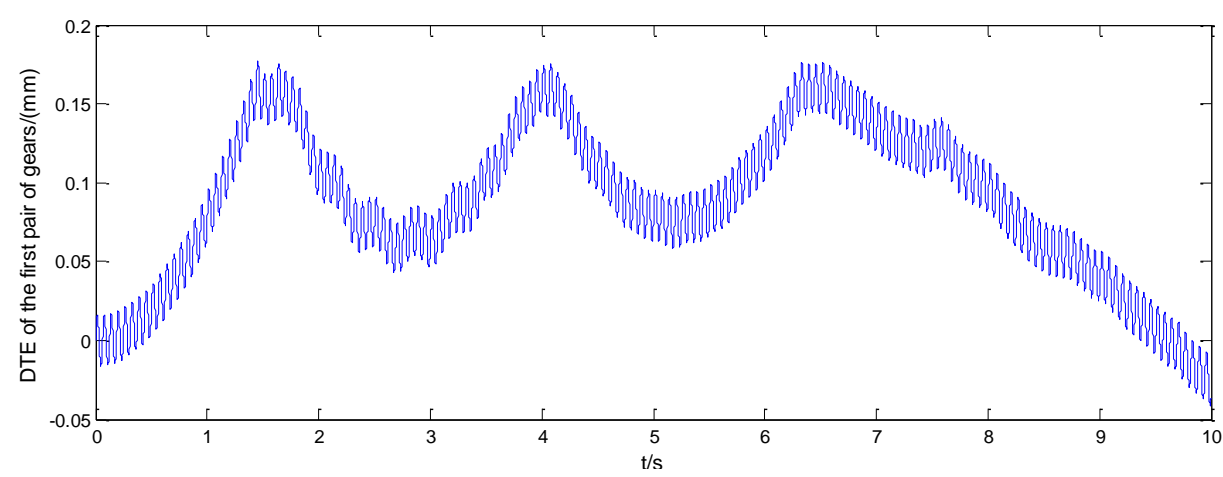

Figure 6. The dynamic transmission error of the first pair of gears at $b_{12}=2.65 \times 10^{-5} \mathrm{~m}$.

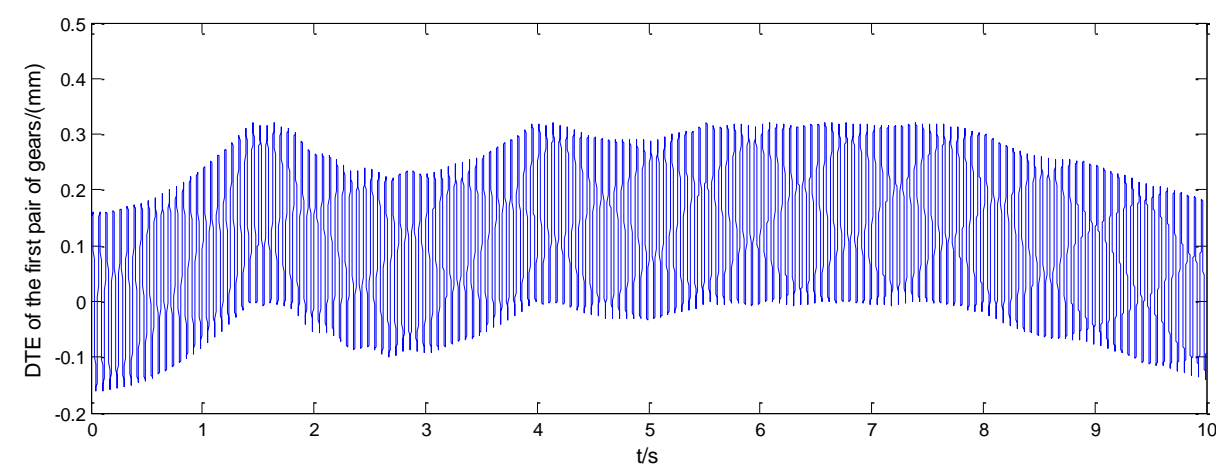

Figure 7. The dynamic transmission error of the first pair of gears at $b_{12}=2.65 \times 10^{-4} \mathrm{~m}$.

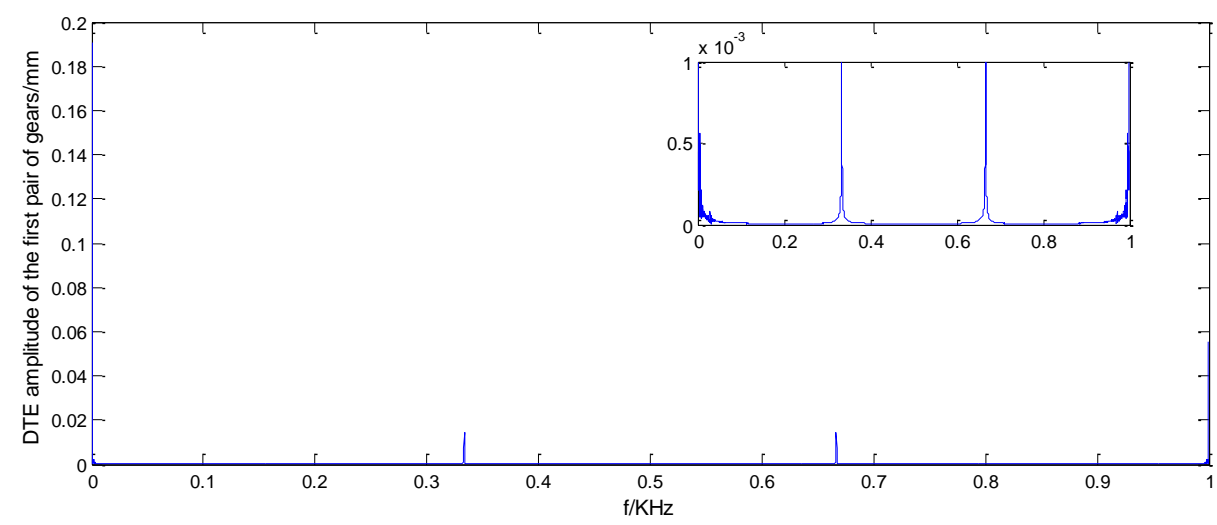

Figure 8. DTE amplitude of the first pair of gears at $\mathrm{n}=1000 \mathrm{r} / \mathrm{min}, \mathrm{b}_{12}=2.65 \times 10^{-5} \mathrm{~m}$. 


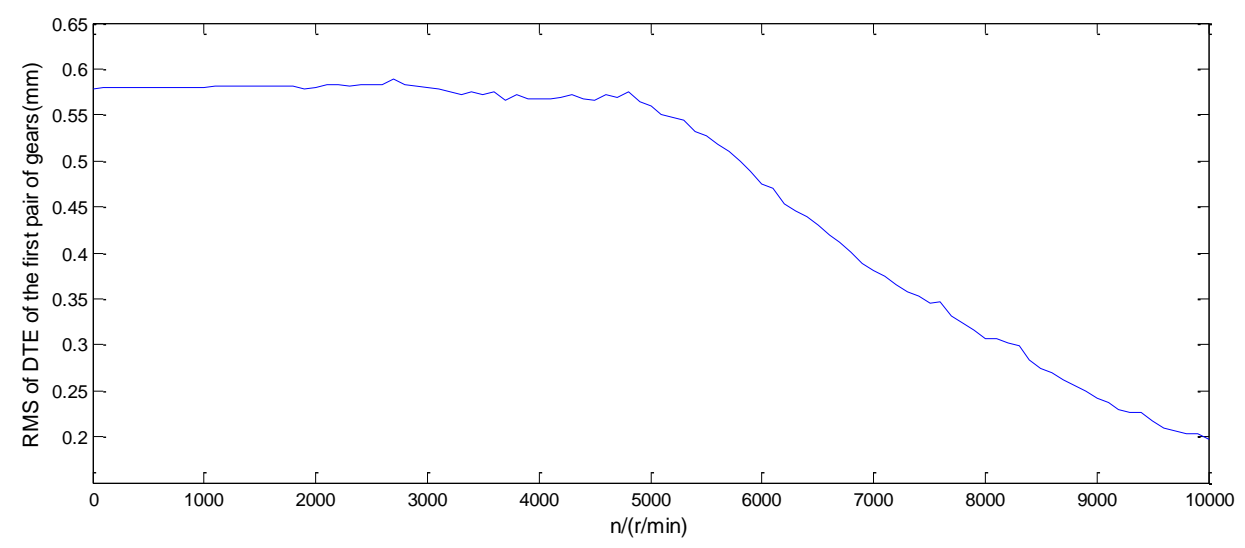

Figure 9. RMS of DTE of the first pair of gears at $b_{12}=2.65 \times 10^{-5} \mathrm{~m}$.

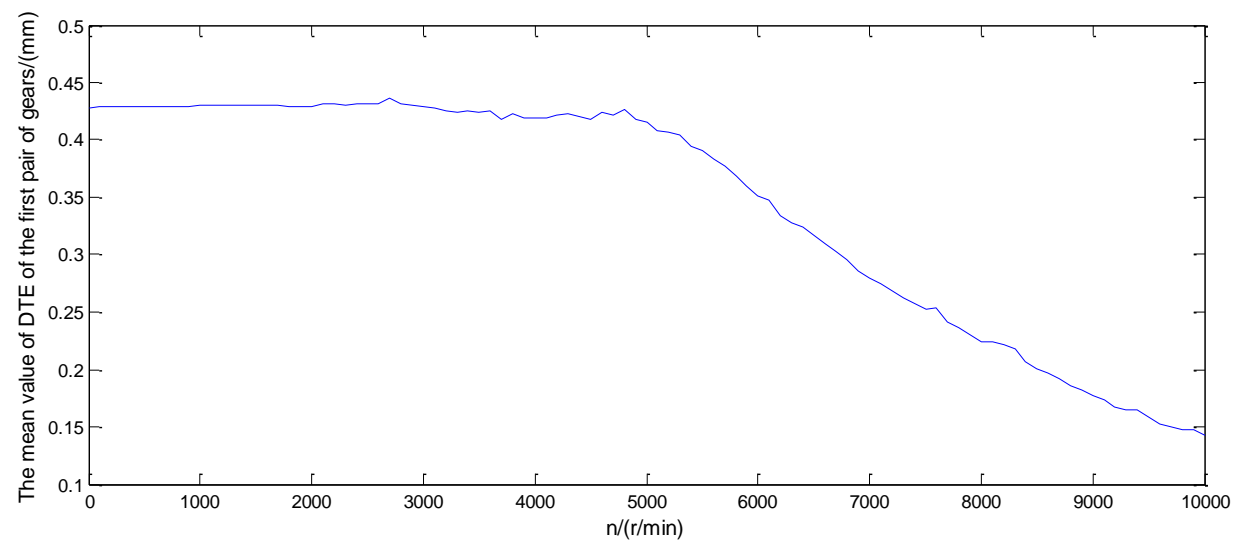

Figure 10. The mean value of DTE of the first pair of gears at $b_{12}=2.65 \times 10^{-5} \mathrm{~m}$.

\subsection{The Analysis about Dynamic Meshing Force of the First Pair of Gears}

The change of dynamic meshing force reflects the magnitude of impact and the intensity of noise for the system. In order to reflect the problem of random effect [32-35], it is assumed that $\mathrm{Cg}_{\Delta}$ obeys the random distribution between zero and one, which is shown in Figure 11. The horizontal coordinate represents the number of samples and the vertical coordinate represents the random distribution value of $\mathrm{Cg}_{\Delta}$ obeying between zero and one. The change of dynamic meshing force at $\mathrm{n}=1000 \mathrm{r} / \mathrm{min}, \mathrm{b}_{12}=2.65 \times 10^{-5} \mathrm{~m}, \mathrm{Cg}_{\Delta}=0$ and $\mathrm{n}=1000, \mathrm{~b}_{12}=2.65 \times 10^{-5} \mathrm{~m}, \mathrm{Cg}_{\Delta}=0.5$ are shown as Figures 12 and 13. From Figures 12 and 13, the fluctuation trend of dynamic meshing force at $\mathrm{n}=1000 \mathrm{r} / \mathrm{min}, \mathrm{b}_{12}=2.65 \times 10^{-5} \mathrm{~m}, \mathrm{Cg}_{\Delta}=0$ and $\mathrm{n}=1000 \mathrm{r} / \mathrm{min}, \mathrm{b}_{12}=2.65 \times 10^{-5} \mathrm{~m}, \mathrm{Cg}_{\triangle}=0.5$ is basically the same. The dynamic meshing force fluctuates greatly at $\mathrm{t}<7 \mathrm{~s}$, while it fluctuates slightly at $\mathrm{t}>7 \mathrm{~s}$ because the system is in a stable state. Compared with Figures 12 and 13, the maximum dynamic meshing force is about $5.9 \mathrm{KN}$ when $\mathrm{T}<7 \mathrm{~s}$ at $\mathrm{n}=1000 \mathrm{r} / \mathrm{min}, \mathrm{b}_{12}=2.65 \times 10^{-5} \mathrm{~m}, \mathrm{Cg}_{\Delta}=0$, while the maximum dynamic meshing force is about $3.8 \mathrm{KN}$ when $\mathrm{T}>7 \mathrm{~s}$. Meanwhile, the maximum dynamic meshing force is about $7.8 \mathrm{KN}$ when $\mathrm{T}<7 \mathrm{~s}$ at $\mathrm{n}=1000 \mathrm{r} / \mathrm{min}, \mathrm{b}_{12}=2.65 \times 10^{-5} \mathrm{~m}, \mathrm{Cg}_{\Delta}=0.5$, while the maximum dynamic meshing force is about $5.5 \mathrm{KN}$ when $\mathrm{T}>7 \mathrm{~s}$. Obviously, the fluctuation of dynamic meshing force increases with the increase of $\mathrm{Cg}_{\Delta}$. 


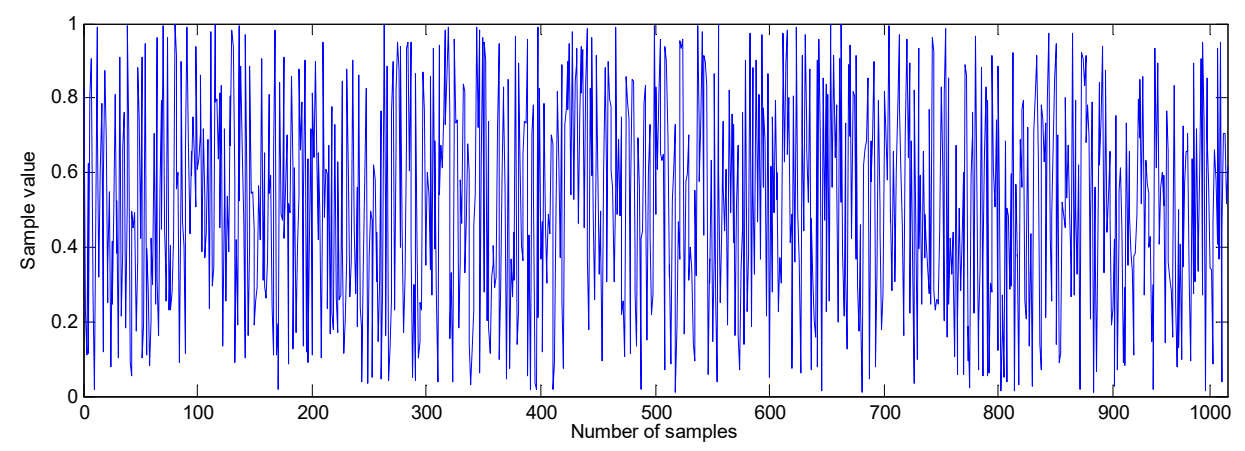

Figure 11. The random distribution of $\mathrm{Cg}_{\triangle}$ obeying between zero and one.

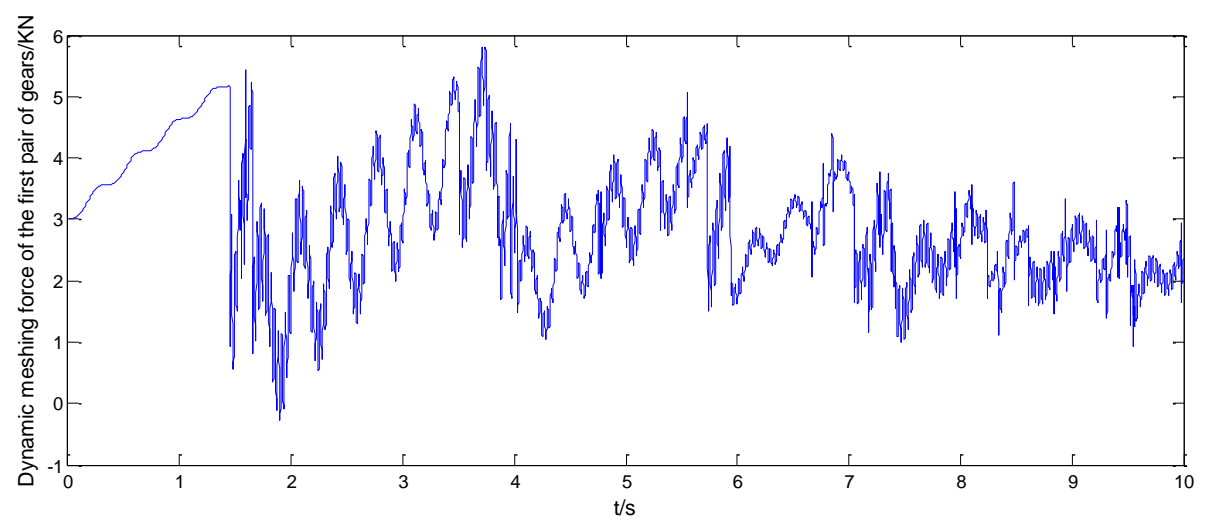

Figure 12. The dynamic meshing force of the first pair of gears at $\mathrm{n}=1000 \mathrm{r} / \mathrm{min}, \mathrm{b}_{12}=2.65 \times 10^{-5} \mathrm{~m}$, $\mathrm{Cg}_{\triangle}=0$.

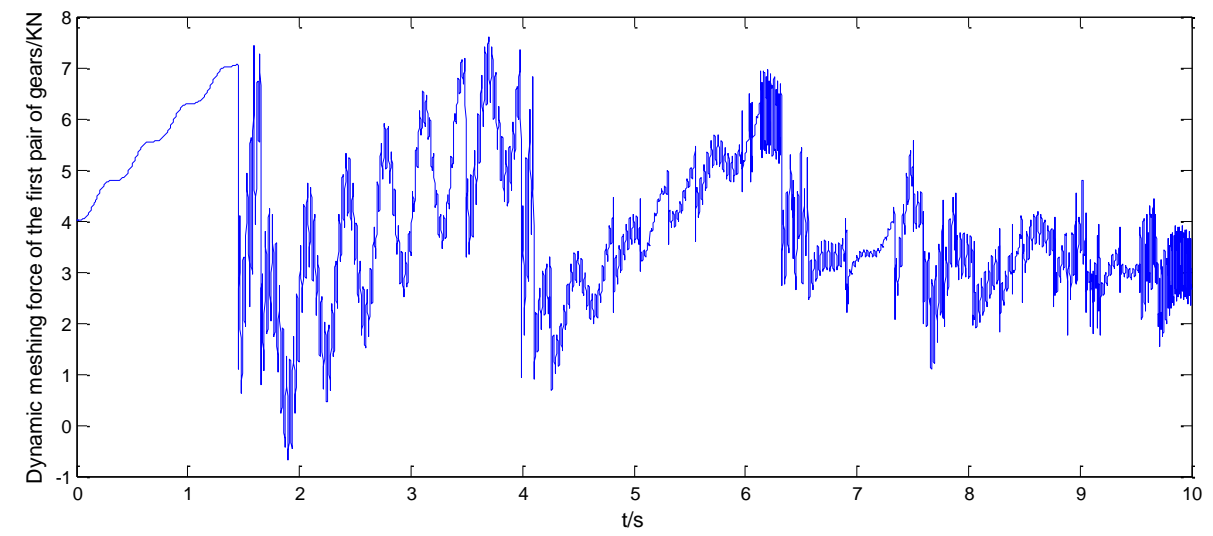

Figure 13. The dynamic meshing force of the first pair of gears at $\mathrm{n}=1000 \mathrm{r} / \mathrm{min}, \mathrm{b}_{12}=2.65 \times 10^{-5} \mathrm{~m}$, $\mathrm{Cg}_{\triangle}=0.5$.

\section{Conclusions}

In this paper, the non-linear dynamic equations of a n-elements closed-loop gear transmission system with symmetrical structure for 3D circular braiding machine including static transmission error, the random disturbance of meshing damping and backlash were presented. The non-linear dynamic feature of multiple-stage closed-loop gear transmission system for the well-known radial braiding machine with one layer was in accordance with practical engineering. The simulation results are summarized as follows: 
1. It is known that the dynamic transmission error will fluctuate with the increase of half-tooth side clearance. Therefore, it is very important to reduce half-tooth side clearance as much as possible to prevent the fluctuation of the dynamic transmission error.

2. It can be seen that the amplitude of dynamic transmission error (DTE) is larger at $325 \mathrm{~Hz}$, $675 \mathrm{~Hz}, 1000 \mathrm{~Hz}$ when $\mathrm{b}_{12}=2.65 \times 10^{-5} \mathrm{~m}, \mathrm{n}=1000 \mathrm{r} / \mathrm{min}$. Since of a constant term of half-tooth side clearance, there is a large amplitude of dynamic transmission error (DTE) at $0 \mathrm{~Hz}$. Since the excessive dynamic displacement caused by the vibration response of the transmission system will affect the normal operation of the mechanical equipment. Therefore, it is very important to avoid $0 \mathrm{~Hz}, 325 \mathrm{~Hz}$, $675 \mathrm{~Hz}, 1000 \mathrm{~Hz}$ when $\mathrm{b}_{12}=2.65 \times 10^{-5} \mathrm{~m}, \mathrm{n}=1000 \mathrm{r} / \mathrm{min}$ to prevent the large amplitude of dynamic transmission error (DTE).

3. The fluctuation trend of RMS and the mean value of DTE under the different speed is basically the same. RMS and the mean value of DTE are stable when the speed is less than $5000 \mathrm{r} / \mathrm{min}$. While RMS and the mean value of DTE are decreasing because of backlash, which can lead to a large proportion of gear separation and lateral contact when the speed is greater than $5000 \mathrm{r} / \mathrm{min}$. Therefore, the speed of $3 \mathrm{D}$ circular braiding machine should be lower than $5000 \mathrm{r} / \mathrm{min}$ to ensure the normal operation of the mechanical equipment.

4. The fluctuation of dynamic meshing force increases with the increase of $\mathrm{Cg}_{\Delta}$. Meanwhile, the amplitude of dynamic meshing force fluctuates greatly at the beginning, and then tends to fluctuate steadily. Therefore, it is very important to reduce $\mathrm{Cg}_{\Delta}$ as much as possible to prevent the fluctuation of dynamic meshing force.

The future perspective of research could be to study the non-linear dynamic feature of multiple-stage closed-loop gear transmission system with other incentive factors for the mechanical equipment in various fields. The influence of different incentive factors on the normal operation of mechanical equipment in various fields.

Author Contributions: L.Y. conceived and performed the experiments and wrote the paper; Z.M. revised and guided the paper; J.B. check and proofread the article; Y.S. contributed the experimental equipment. All authors have read and agreed to the published version of the manuscript.

Funding: The Special Fund of Scientific and Technological Achievements Transformation in Jiangsu Province (Grant Nos. BA2018061). The Program of Jiangsu Innovative Talent (Grant Nos. 20171122). The Fundamental Research Funds for the Central Universities and Graduate Student Innovation Fund of Donghua University (Grant Nos. CUSF-DH-D-2019097).

Acknowledgments: The authors would like to thank the anonymous reviewers for their valuable comments and suggestions to improve the paper.

Conflicts of Interest: The authors declared no potential conflict of interest with respect to the research, authorship and/or publication of this article.

\section{References}

1. Megel, M.; Kumosa, L.; Ely, T.; Armentrout, D.; Kumosa, M. Initiation of stress-corrosion cracking in unidirectional glass/polymer composite materials. Compos. Sci. Technol. 2001, 61, 231-246. [CrossRef]

2. Hull, D.; Kumosa, M.; Price, J.N. Stress corrosion of aligned glass fiber-polyester composite material. Mater. Sci. Tech. Lond. 1985, 1, 177-182. [CrossRef]

3. Noble, B.; Harris, S.J.; Owen, M.J. Stress corrosion cracking of GRP pultruded rods in acid environments. J. Mater. Sci. 1983, 18, 1244-1254. [CrossRef]

4. Ma, G.; Branscomb, D.; Beale, D.G. Modeling of the tensioning system on a braiding machine carrier. Mech. Mach. Theory 2012, 47, 46-61. [CrossRef]

5. Zhou, H.; Zhang, W.; Liu, T.; Gu, B.; Sun, B. Finite element analyses on transverse impact behaviors of 3-D circular braided composite tubes with different braiding angles. Compos. Part A Appl. Sci. Manuf. 2015, 79, 52-62. [CrossRef]

6. Guyader, G.; Gabor, A.; Hamelin, P. Analysis of 2D and three-dimensional circular braiding processes: Modeling the interaction between the process parameters and the pre-form architecture. Mech. Mach. Theory 2013, 69, 90-104. [CrossRef] 
7. Van Ravenhorst, J.; Akkerman, R. Circular braiding take-up speed generation using inverse kinematics. Compos. Part A Appl. Sci. Manuf. 2014, 64, 147-158. [CrossRef]

8. Van Ravenhorst, J.; Akkerman, R. A yarn interaction model for circular braiding. Compos. Part A Appl. Sci. Manuf. 2016, 81, 254-263. [CrossRef]

9. Na, W.; Ahn, H.; Jeon, S.; Lee, J.S.; Kang, H.; Yu, W. Prediction of the braid pattern on arbitrary-shaped mandrels us-ing the minimum path condition. Compos. Sci. Technol. 2014, 91, 30-37. [CrossRef]

10. Hans, T.; Cichosz, J.; Brand, M.; Hinterhölzl, R. Finite element simulation of the braiding process for arbitrary mandrel shapes. Compos. Part A Appl. Sci. Manuf. 2015, 77, 124-132. [CrossRef]

11. Kyosev, Y. Braiding Technology for Textiles; Woodhead Publishing: MÖnchengladbach, Germany, 2015.

12. Philippe, M.; Jonathan, L.; Lebel, L.L. Automated braiding of a complex aircraft fuselage fr-ame using a non-circular braiding model. Compos. Part A Appl. Sci. 2017, 102, 48-63.

13. Heieck, F.; Hermann, F.; Middendorf, P.; Schladitz, K. Influence of the cover factor of 2D biaxial and triaxial braided carbon composites on their in-plane mechanical properties. Compos. Struct. 2017, 163, 114-122. [CrossRef]

14. Wehrkamp-Richter, T.; Hinterhölzl, R.; Pinho, S.T. Damage and failure of triaxial braided composites under multi-axial stress states. Compos. Sci. Technol. 2017, 150, 32-44. [CrossRef]

15. Swery, E.; Hans, T.; Bultez, M.; Wijaya, W.; Kelly, P.; Hinterhölzl, R.; Bickerton, S. Complete simulation process chain for the manufacturing of braided composite parts. Compos. Part A Appl. Sci. Manuf. 2017, 102, 378-390. [CrossRef]

16. Kahraman, A.; Singh, R. Non-linear dynamics of a spur gear pair. J. Sound Vib. 1990, 142, 49-75. [CrossRef]

17. Kahraman, A.; Singh, R. Interactions between time-varying mesh stiffness and clearance non-linearities in a geared system. J. Sound Vib. 1991, 146, 135-156. [CrossRef]

18. He, S.; Rook, T.; Singh, R. Construction of semianalytical solutions to spur gear dyna mics given periodic mesh stiffness and sliding friction functions. J. Mech. Design 2008, 130, 1226011-1226019. [CrossRef]

19. Liu, G.; Parker Robert, G. Dynamic modeling and analysis of tooth profile modification for multi-mesh gear vibration. J. Mech. Design 2008, 130, 1214021-12140213. [CrossRef]

20. Cui, Y.; Liu, Z.; Wang, Y.; Ye, J. Nonlinear Dynamic of a Geared Rotor System with Nonlinear Oil Film Force and Nonlinear Mesh Force. J. Vib. Acoust. 2012, 134, 041001. [CrossRef]

21. Baguet, S.; Jacquenot, G. Nonlinear couplings in a gear-shaft-bearing system. Mech. Mach. Theory 2010, 45, 1777-1796. [CrossRef]

22. Li, S.; Kahraman, A. A tribo-dynamic model of a spur gear pair. J. Sound Vib. 2013, 332, 4963-4978. [CrossRef]

23. Wei, J.; Sun, Q.; Sun, W.; Qin, D.; Zhu, C.; Zhu, W.; Guo, A. Dynamic analysis and effects of nonlinear factors of a gear transmission system for high speed locomotive. J. Vib. Shock 2012, 31, 38-43, 50.

24. Zhang, H.; Wang, R.; Chen, Z.; Wei, C.; Zhao, Y.; You, B.; School of Astronautics, Harbin Institute of Technology. Nonlinear dynamic analysis of a gear-rotor system with coupled multi-clearance. J. Vib. Shock 2015, 34, 144-150, 192.

25. Li, R.; Wang, J. Gear System Dynamics-Vibration, Shock \& Nois; Science Press: Beijing, China, 1997; pp. $158-163$.

26. Zhang, Y. Mechanical vibration; Tsinghua University Press: Beijing, China, 2007; pp. 78-100.

27. Al-Shyyab, A.; Kahraman, A. Non-linear dynamic analysis of a multi-mesh gear train using multi-term harmonic balance method: Period-one motions. J. Sound Vib. 2005, 284, 151-172. [CrossRef]

28. Yi, Y.; Huang, K.; Xiong, Y.; Sang, M. Non-linear dynamic modelling and analysis for a spur gear system with time-varying pressure angle and gear backlash. Mech. Syst. Signal Process. 2019, 132, 18-34. [CrossRef]

29. Özgüven, H.N.; Houser, D. Mathematical models used in gear dynamics-A review. J. Sound Vib. 1988, 121, 383-411. [CrossRef]

30. Shin, D.; Palazzolo, A. Nonlinear analysis of a geared rotor system supported by fluid film journal bearings. J. Sound Vib. 2020, 475, 115269. [CrossRef]

31. Pan, W.; Li, X.; Wang, L.; Yang, Z. Non-linear response analysis of gear-shaft-bearing system considering tooth contact temperature and random excitations. Appl. Math. Model 2019, 68, 113-136. [CrossRef]

32. Hasnijeh, S.G.; Poursina, M.; Leira, B.J.; Karimpour, H.; Chai, W. Stochastic dynamics of a nonlinear time-varying spur gear model using an adaptive time-stepping path integration method. J. Sound Vib. 2019, 447, 170-185. [CrossRef]

33. Wang, Y.; Zhang, W.J. Stochastic vibration model of gear transmission systems con-sidering speed-dependent random errors. Nonlinear Dyn. 1998, 17, 187-203. [CrossRef] 
34. Naess, A.; Kolnes, F.E.; Mo, E. Stochastic spur gear dynamics by numerical path in-tegration. J. Sound. Vib. 2007, 302, 936-950. [CrossRef]

35. Fang, Y.; Liang, X.; Zuo, M.J. Effects of friction and stochastic load on transient cha-racteristics of a spur gear pair. Nonlinear Dyn. 2018, 93, 599-609. [CrossRef]

Publisher's Note: MDPI stays neutral with regard to jurisdictional claims in published maps and institutional affiliations.

(C) 2020 by the authors. Licensee MDPI, Basel, Switzerland. This article is an open access article distributed under the terms and conditions of the Creative Commons Attribution (CC BY) license (http://creativecommons.org/licenses/by/4.0/). 